\section{Correlation in foveal thickness \\ measurements \\ between \\ spectral-domain and \\ time-domain optical \\ coherence \\ tomography in \\ normal individuals}

\begin{abstract}
Purpose To compare a time-domain (Stratus) and a spectral-domain (Spectralis) optical coherence tomography (OCT) device in assessing foveal thickness in healthy subjects. Methods In this observational study 40 healthy subjects (40 eyes) underwent Stratus OCT and Spectralis OCT measurements of foveal thickness using three consecutive horizontal and vertical B-scan. Paired samples $t$-test was used to compare means between Stratus and Spectralis OCT measurements. Coefficient of variation ( $\mathrm{CoV}$ ) was used to compare dispersion in datasets. Pearson's correlation coefficient was used to quantify linear relation between Spectralis and Stratus OCT measurements. To assess agreement between Spectralis and Stratus OCT foveal thickness measurements, the Bland and Altman plots were used.

Results Sample age ranged from 19 to 49 years (mean 33.25, standard deviation (SD) \pm 4.22).

The Spectralis OCT foveal thickness measurements resulted significantly higher than those obtained with Stratus OCT $(227.64 \pm 11.74$ vs $144.36 \pm 12.25 \mu \mathrm{m}$, and $227.63 \pm 11.43$ vs $144.92 \pm 12.34 \mu \mathrm{m}$, for horizontal and vertical foveal thickness, respectively) $(P<0.05)$. Coefficient of variations were 5.16 and $5.02 \%$ using Spectralis OCT, and 8.49 and $8.51 \%$ using Stratus OCT. Mean Spectralis/Stratus ratio was 1.58 for both horizontal and vertical measurements. A linear relation between the
\end{abstract}

P Carpineto, M Nubile, L Toto, A Aharrh Gnama, L Marcucci, L Mastropasqua and M Ciancaglini two technologies was found $\left(r_{\text {horiz }}=0.899\right.$ and $\left.r_{\text {vert }}=0.869\right)(P<0.001)$.

Conclusions A good correlation between Stratus and Spectralis OCT foveal measurements was found, independently of retinal thickness. This preliminary study suggests the existence of a conversion factor between Stratus and Spectralis OCT when measuring healthy foveal thickness.

Eye (2010) 24, 251-258; doi:10.1038/eye.2009.76; published online 24 April 2009

Keywords: optical coherence tomography; time domain; spectral domain; macular thickness

\section{Introduction}

Optical coherence tomography (OCT) is a well-established noninvasive technique for the imaging of the macula, capable of monitoring the effects of medical and surgical interventions in macular diseases by measuring the retinal thickness. ${ }^{1,2}$

Imaging speed in standard OCT systems is limited by the time-domain (TD) detection technique, which measures the echo time delay of back-scattered or back-reflected light through an interferometer with a mechanically scanning optical reference path. ${ }^{1}$ This instrument acquires cross-sectional images consisting of $512 \mathrm{~A}$-scans in $1.28 \mathrm{~s}$. The axial resolution of TD-OCT is $\sim 8-10 \mu \mathrm{m}$.

Recently, acquisition speed and sensitivity have been improved by a novel detection
Department of Medicine and Aging Sciences, Section of Ophthalmology, University 'G. d'Annunzio', Chieti-Pescara, Chieti, Italy

Correspondence:

P Carpineto,

Department of Medicine and Aging Sciences, Section of Ophthalmology, University G. d'Annunzio Chieti-Pescara,

Via dei Vestini 21, Chieti 66100,

Italy

Tel: + 390871358 489; Fax: + 390871358 794;

E-mail: p.carpineto@ unich.it

Received: 4 November 2008 Accepted in revised form: 13 March 2009 Published online: 24 April 2009

Conflict of interest: None. Proprietory interest: None. 
technique known as spectral-domain (SD), Fourier domain or frequency-domain detection, which enables a 25- to 100-fold increase in imaging speed over standard ophthalmic OCT systems. ${ }^{3-7}$

In SD detection, the echo time delays of light are measured by Fourier transforming the interference spectrum of the light signal, requiring no mechanical axial scanning, and this results in a higher acquisition speed with respect to TD-OCT. In addition, it has been theoretically predicted and experimentally confirmed that SD-OCT has a higher sensitivity than that of TD-OCT. ${ }^{4,8}$ Practically, the SD-OCT method shortens the examination time and thereby reduces eye exposure and image-blurring artifacts. ${ }^{6,9}$

The Spectralis OCT is a SD-OCT system that allows high-speed, high-resolution cross-sectional imaging of the retina, with simultaneous OCT and infrared reflectance imaging.

To date TD-OCT still has widespread diffusion, and can be considered as the 'gold standard' technique for retinal thickness measurements. Therefore, every OCT technique having the aim to measure retinal thickness has to be compared with TD-OCT before obtaining scientific and clinical consensus.

The purpose of our study was to measure the foveal thickness in healthy participants with both TD- and SDOCT, to compare data, and to detect differences between the two retinal measurements techniques.

\section{Materials and methods}

The study was carried out at the Ophthalmology Clinic of the University of Chieti-Pescara, Italy. Healthy participants with an age between 19 and 50 years were enrolled in the study. The study adhered to the tenets of the declaration of Helsinki and was approved by the local ethical committee. Written informed consent was obtained from all the subjects. Each participant underwent a comprehensive ophthalmic examination, including review of the medical history, best corrected visual acuity assessment, slit-lamp biomicroscopy, intraocular pressure measurement with Goldmann applanation tonometry, gonioscopy, and dilated funduscopic examination using a 78-D lens. To be included, participants had to have best-corrected visual acuity of $20 / 25$ or better, spherical refraction within $\pm 3.0 \mathrm{D}$, cylinder correction within $\pm 2.0 \mathrm{D}$, open angles on gonioscopy, intraocular pressures of $21 \mathrm{~mm} \mathrm{Hg}$ or less, a healthy appearance of the macula lutea, and the optic disc and RNFL (no diffuse or focal rim thinning cupping, optic disc haemorrhage, or RNFL defects), as evaluated by funduscopic examination.
Patients were excluded if they had any earlier history of ocular disease, surgery or treatment, or any systemic disease with ocular involvement.

Eyes with corneal or lens opacities, retinal disease, uveitis, glaucomatous or nonglaucomatous optic neuropathy were excluded from the investigation.

In the same session, in one randomly selected eye of each participant, three consecutive measurements of foveal thickness were carried out using both TD-OCT and SD-OCT. All scans were carried out by the same examiner (AAG), who had been trained on both OCT devices.

Time-domain optical coherence tomography images were obtained from each eye after pupil dilation, using the Stratus OCT system (Carl Zeiss Meditec Inc., Dublin, CA, USA). The same OCT version (4.1) was used for the duration of the study.

The Stratus OCT thickness algorithm calculates retinal thickness as the distance between the vitreo-retinal interface and the anterior surface of the retinal pigment epithelium.

The line scan protocol was selected to acquire three consecutive horizontal $\left(0^{\circ}\right)$ and vertical $\left(270^{\circ}\right)$ B-scans passing through the fovea (six scans in total). Scan length was set at $5 \mathrm{~mm}$ (Figure 1). The retinal thickness analysis protocol provided with the instrument software was used to calculate the foveal thickness. The values obtained from the three horizontal and the three vertical scans were averaged for statistical analysis.

Before the examination a signal to noise ratio $\geqslant 25 \mathrm{~dB}$ and a good quality A-scans $\geqslant 90 \%$ were preimposed. The retinal thickness analysis software automatically displays the signal strength. A signal strength $\geqslant 7$ was used as objective quality control. In addition, only scans with no interfering noise or artifacts were included in the study, and each individual scan was reviewed for aberrant placement of the inner and outer retinal borders.

Spectral-domain optical coherence tomography images were obtained using the HRA-OCT Spectralis unit (Heidelberg Engineering $\mathrm{GmbH}$, Heidelberg, Germany).

The Spectralis OCT uses SD-OCT technology, also referred to as Fourier domain OCT. The beam of a super luminescence diode (SLD) scans across the retina to produce a cross-sectional B-scan image. The infrared beam of the SLD has an average wavelength of $870 \mathrm{~nm}$.

The Spectralis OCT system achieves data acquisition rate of up to 40000 axial scans per second, with axial scan depth of $1.8 \mathrm{~mm}$.

The axial optical resolution, determined by the bandwidth of the SLD light source, the design of the spectrometer (optic and detector), and the numerical aperture of the optic, is calculated to be $7 \mu \mathrm{m}$. 

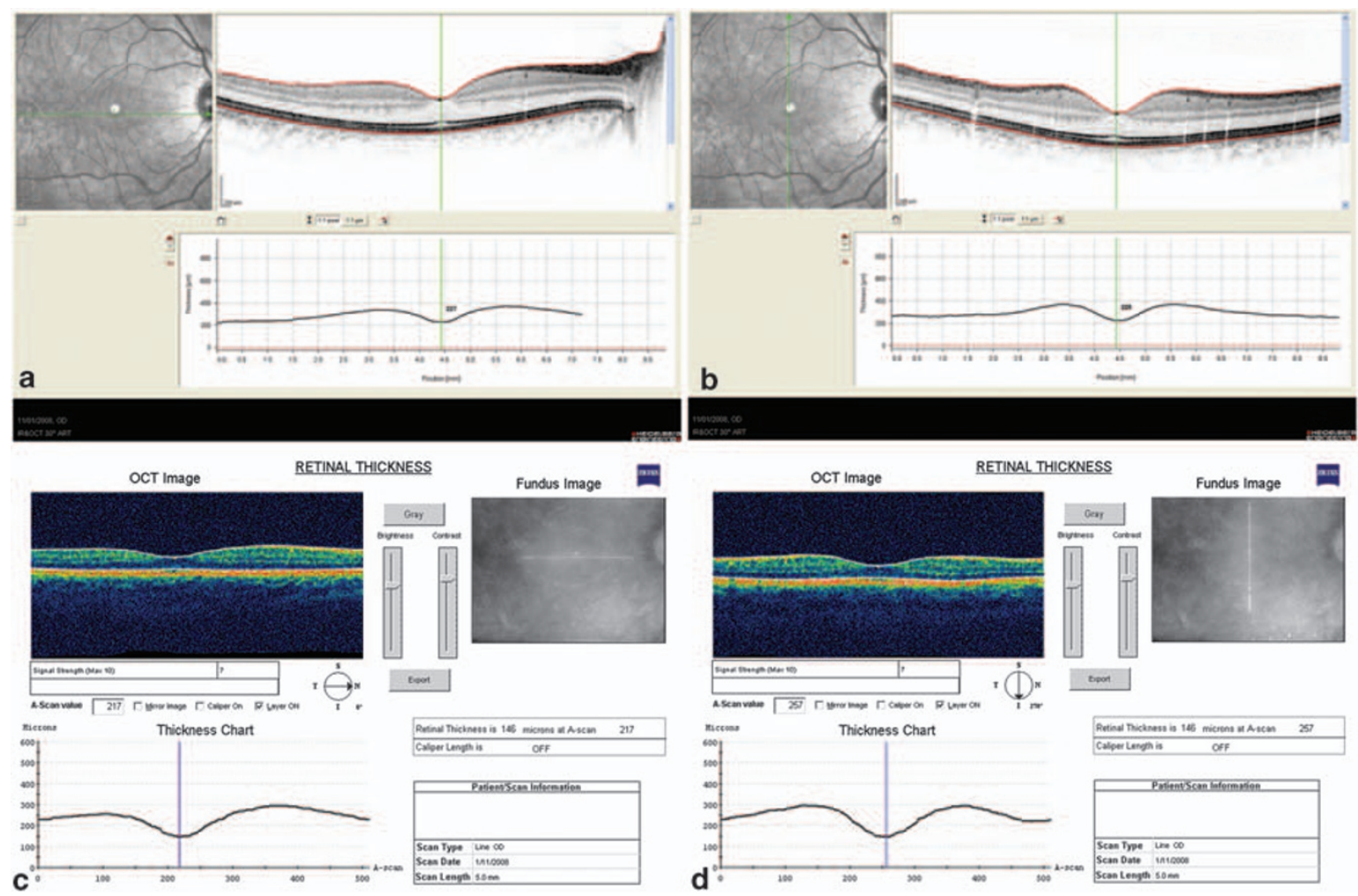

Figure 1 Horizontal (a-top left) and vertical (b-top right) Spectralis foveal thickness measurements. Horizontal (c-bottom left) and vertical (d-bottom right) Stratus foveal thickness measurements.

Optical lateral resolution is $14 \mu \mathrm{m}$, which corresponds to a $7 \mu \mathrm{m}$ spacing according to Nyquist's sampling theorem.

In the High Speed Mode, the Spectralis OCT acquires 48 B-scans (768 axial scans/transverse pixels in each image) per second. Acquisition time is $19 \mathrm{~ms} / \mathrm{B}$-scan and lateral resolution is $11 \mu \mathrm{m} /$ pixel.

In the High Resolution Mode, the digital scan rate is 25 B-scans per second (1536 axial scans/transverse pixels in each image). Acquisition time is $38 \mathrm{~ms} / \mathrm{B}-\mathrm{Scan}$ and lateral resolution is $6 \mu \mathrm{m} /$ pixel digital.

For retinal thickness measurements, the High Speed Mode was used, with a B-scan angle of $30^{\circ}$.

The section scan option was used to acquire three consecutive horizontal and vertical B-scans passing through the fovea (total of six scans). The scan length was $9 \mathrm{~mm}$ by default (Figure 1). The retinal thickness analysis option was used to calculate the foveal thickness.

The Spectralis OCT thickness algorithm calculates retinal thickness as the distance between the vitreoretinal interface and the Bruch's membrane. Similarly to the protocol used for TD-OCT, the values obtained from horizontal and vertical scans were averaged for statistical analysis.
The authors certify that all applicable institutional and governmental regulations concerning the ethical use of human volunteers were followed during this research.

\section{Statistical analysis}

All data were analysed with the statistical package MedCalc version 8.1 for Windows (MedCalc, Mariakerke, Belgium).

The D'Agostino-Pearson's test that computes a single $P$-value for the combination of the coefficients of Skewness and Kurtosis was used to study the sample distribution.

Paired samples $t$-test was used to compare means between Stratus and Spectralis measurements, and horizontal and vertical measurements.

Pearson's correlation coefficient was used to quantify linear relation between the Spectralis and the Stratus OCT measurements.

The $\mathrm{CoV}$ was used to compare the degree of variation from one data series to another. Standard deviation (SD) has little interpretable meaning on its own unless the mean value is also reported alongwith. For a given SD 
value, it indicates a high or low degree of variability, only in relation to the mean value. For this reason, the variability of a distribution of data is better described dividing the SD by the mean. The representation of the obtained value as a \% of mean gives the $\mathrm{CoV}$. The $\mathrm{CoV}$ is particularly useful when comparing dispersion in datasets with markedly different means; it is only defined for nonzero mean, and it is most useful for variables that are always positive. The $\mathrm{CoV}$ should only be computed for data measured on a ratio scale. ${ }^{10}$

To assess the agreement between Spectralis and Stratus OCT foveal measurements the Bland and Altman correlation plots for foveal thickness values were used. ${ }^{11}$ For both horizontal and vertical foveal measurements, the ratio (Spectralis/stratus OCT foveal thickness) was plotted against the average of the two values. The ratio option is useful when the researcher hypothesises an increase in the variability of the differences as the magnitude of the measurement increases. ${ }^{12}$

\section{Results}

Forty eyes of 40 participants underwent foveal thickness measurements with the earlier described protocols of the two OCT devices (Table 1). In all the eyes included in the study high-quality scans were obtained. The sample age ranged from 19 to 49 years (mean 33.25, $\mathrm{SD} \pm 4.22$ ).

The distribution of foveal thickness measurements was classified as normal using both TD- and SD-OCT technologies.

Mean TD-OCT foveal thickness was $144.36 \mu \mathrm{m}$ (SD $\pm 12.25 \mu \mathrm{m}$ ) using horizontal line, and $144.92 \mu \mathrm{m}$ $(\mathrm{SD} \pm 12.34 \mu \mathrm{m}$ ) using vertical line (paired samples $t$-test $P=0.408$ ). CoVs were 8.49 and $8.51 \%$ using horizontal and vertical line, respectively.

Mean SD-OCT foveal thickness was $227.64 \mu \mathrm{m}$ (SD $\pm 11.74 \mu \mathrm{m}$ ) using horizontal line, and $227.63 \mu \mathrm{m}$ $(\mathrm{SD} \pm 11.43 \mu \mathrm{m})$ using vertical line (paired samples $t$-test $P=0.990$ ). CoVs were 5.16 and $5.02 \%$, respectively.

For both horizontal and vertical foveal thickness, paired samples $t$-test showed a statistically significant difference between the Stratus and Spectralis OCT measurements $(P=0.000)$.

Figure 2 shows the Bland and Altman plot of the agreement in horizontal foveal thickness between TDand SD-OCT assessment. The ratio (SD-OCT horizontal foveal thickness/TD-OCT horizontal foveal thickness) is plotted against the average of the two measurements.

Mean SD-OCT/TD-OCT ratio for horizontal measurements was 1.58 (range 1.42-1.75).

The existence of a linear relation between SD- and TDOCT horizontal measurements is indicated by Pearson's correlation coefficient $(r=0.899 ; P<0.001)$ (Figure 3).
Figure 4 shows the Bland and Altman plot of the agreement in vertical foveal thickness between SD- and TD-OCT assessment. The ratio (SD-OCT vertical foveal thickness/TD-OCT vertical foveal thickness) is plotted against the average of the two measurements.

Mean SD-OCT/TD-OCT ratio for vertical measurements was 1.58 (range 1.47-1.80).

The existence of a linear relation between SD- and TD-OCT vertical measurements is indicated by Pearson's correlation coefficient $(r=0.869 ; P<0.001)$ (Figure 5).

\section{Discussion}

Optical coherence tomography is a well-established, noncontact, noninvasive, and safe technique, capable of visualising retinal structure and studying macular pathologies. Earlier studies highlighted the reliability of retinal thickness measurements obtained by means of TD-OCT technology. ${ }^{13-15}$ The technique relies on an examination of the light scattered or reflected back from the internal structures of the retina. In TD-OCT, an optical delay line has to be mechanically scanned to record a single line (A-scan) in a tomographic image. ${ }^{1}$

Time-domain OCT takes $1.28 \mathrm{~s}$ to obtain a single crosssectional image consisting of 512 optical axial scans. This acquisition time of TD-OCT, longer than $1 \mathrm{~s}$, may induce motion artifacts in the obtained cross-sectional images. These artifacts can be corrected by automated numerical alignment of adjacent optical A-scans, but this procedure can generate errors in the presence of discontinuities in the retinal structure. ${ }^{16}$

In SD detection, echo time delays of light are measured by Fourier transforming the interference spectrum of the light signal, which requires no mechanical axial scanning, and this results in a 25- to 100-fold higher acquisition speed than that of TD-OCT. The SD-OCT method shortens the examination time and thereby reduces eye exposure as well as image blurring artifacts. ${ }^{6,9}$

Optical coherence tomography foveal thickness measurement is one of the most reliable parameter when studying and following the course of macular pathologies. The progressive introduction into the clinical use of SD-OCT as an alternative to TD-OCT obviously generates questions on the agreement in foveal thickness measurements between the two different OCT technologies.

In this observational, case series study evaluating and comparing two different diagnostic technologies, mean foveal thickness was found to be significantly higher when using SD-OCT. This result confirms the recent data reported by Legarreta et al, ${ }_{1}^{17}$ who compared macular thickness of 13 participants scanned with both a prototype Cirrus HD-OCT (Carl Zeiss Meditec Inc.) and Stratus OCT, and found that the thickness measurements 
Table 1 Stratus and spectralis OCT measurements of foveal thickness

\begin{tabular}{|c|c|c|c|c|}
\hline \multirow[t]{2}{*}{ Participant no. } & \multicolumn{2}{|c|}{ Stratus OCT measurements } & \multicolumn{2}{|c|}{ Spectralis OCT measurements } \\
\hline & Horizontal & Vertical & Horizontal & Vertical \\
\hline 1 & 117 & 115 & 203 & 206 \\
\hline 2 & 156 & 154 & 222 & 219 \\
\hline 3 & 134 & 134 & 224 & 227 \\
\hline 4 & 140 & 140 & 228 & 227 \\
\hline 5 & 152 & 156 & 231 & 233 \\
\hline 6 & 130 & 136 & 221 & 230 \\
\hline 7 & 146 & 146 & 220 & 220 \\
\hline 8 & 160 & 160 & 247 & 242 \\
\hline 9 & 142 & 142 & 225 & 226 \\
\hline 10 & 148 & 146 & 230 & 223 \\
\hline 11 & 136 & 142 & 220 & 221 \\
\hline 12 & 154 & 154 & 234 & 244 \\
\hline 13 & 154 & 154 & 235 & 233 \\
\hline 14 & 142 & 142 & 225 & 231 \\
\hline 15 & 146 & 140 & 216 & 213 \\
\hline 16 & 166 & 166 & 250 & 243 \\
\hline 17 & 152 & 156 & 239 & 235 \\
\hline 18 & 152 & 156 & 235 & 238 \\
\hline 19 & 130 & 132 & 209 & 201 \\
\hline 20 & 132 & 128 & 222 & 219 \\
\hline 21 & 119 & 121 & 213 & 208 \\
\hline 22 & 144 & 144 & 223 & 223 \\
\hline 23 & 134 & 136 & 216 & 220 \\
\hline 24 & 138 & 138 & 227 & 221 \\
\hline 25 & 154 & 148 & 233 & 235 \\
\hline 26 & 132 & 136 & 224 & 223 \\
\hline 27 & 142 & 146 & 225 & 227 \\
\hline 28 & 173 & 169 & 257 & 254 \\
\hline 29 & 138 & 140 & 216 & 219 \\
\hline 30 & 138 & 138 & 218 & 225 \\
\hline 31 & 144 & 144 & 221 & 224 \\
\hline 32 & 150 & 154 & 233 & 234 \\
\hline 33 & 154 & 154 & 233 & 233 \\
\hline 34 & 138 & 140 & 221 & 222 \\
\hline 35 & 126 & 126 & 210 & 210 \\
\hline 36 & 166 & 169 & 250 & 249 \\
\hline 37 & 146 & 146 & 230 & 228 \\
\hline 38 & 154 & 154 & 240 & 242 \\
\hline 39 & 123 & 125 & 207 & 213 \\
\hline 40 & 130 & 134 & 220 & 220 \\
\hline Mean $( \pm$ SD) $\mu \mathrm{m}$ & $144.36( \pm 12.25)$ & $144.92( \pm 12.34)$ & $227.64( \pm 11.74)$ & $227.63( \pm 11.43)$ \\
\hline $\mathrm{CoV} \%$ & 8.49 & 8.51 & 5.16 & 5.02 \\
\hline
\end{tabular}

$\mathrm{CoV}=$ coefficient of variation; $\mathrm{SD}=$ standard deviation.

Each value is the mean of three consecutive measurements.

by using HD-OCT were $\sim 50 \mu \mathrm{m}$ higher than those obtained by Stratus OCT. Similarly, Leung et $a{ }^{18}$ in their study comparing macular thickness measurements obtained by means of Stratus OCT and SD-OCT (3D OCT, Topcon, Tokyo, Japan) in 35 healthy normal participants, found that the foveal and total macular thicknesses measured by SD-OCT were significantly greater than those measured by Stratus OCT (both with $P<0.001$ ). The spans of $95 \%$ limits of agreement for foveal and total macular thicknesses were 33.9 and $21.3 \mu \mathrm{m}$, respectively. More recently, the study by Forte et $a{ }^{19}{ }^{19}$ who compared retinal thickness and volume measurements obtained with Stratus OCT and SD scanning laser ophthalmoscope OCT (SLO/OCT, OTI, Toronto, ON, Canada), confirmed that the macular retinal thickness values measured with SD-SLO/OCT were significantly higher than those measured with Stratus OCT by 30.1-39.2 $\mu \mathrm{m}$.

These results may be related both to the different technology and to the different thickness algorithm used. The Stratus OCT algorithm identifies differences in the image reflectance patterns in each A-scan that compose one tomogram (B-scan), and assumes that the distance 


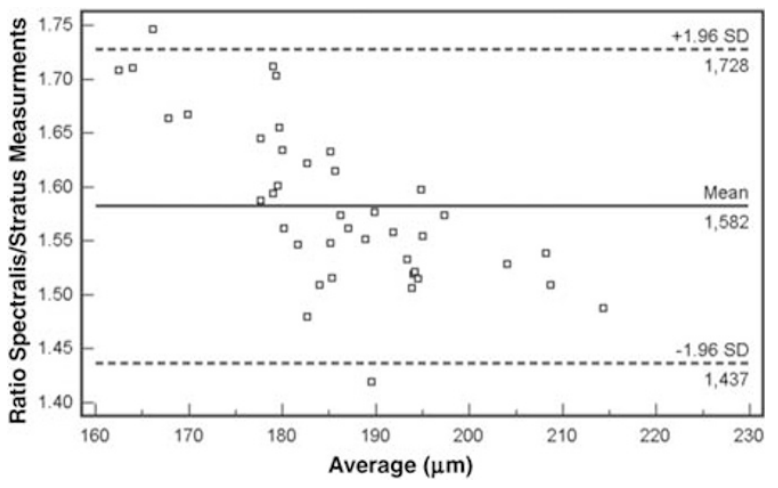

Figure 2 The Bland and Altman plot of the agreement in horizontal foveal thickness between Spectralis and Stratus optical coherence tomography assessment. The ratio between Spectralis horizontal foveal thickness and Stratus horizontal foveal thickness is plotted against the average of the two measurements. Horizontal lines are drawn at the mean ratio, and at the mean ratio plus and minus 1.96 times the standard deviation (SD) of the ratios.

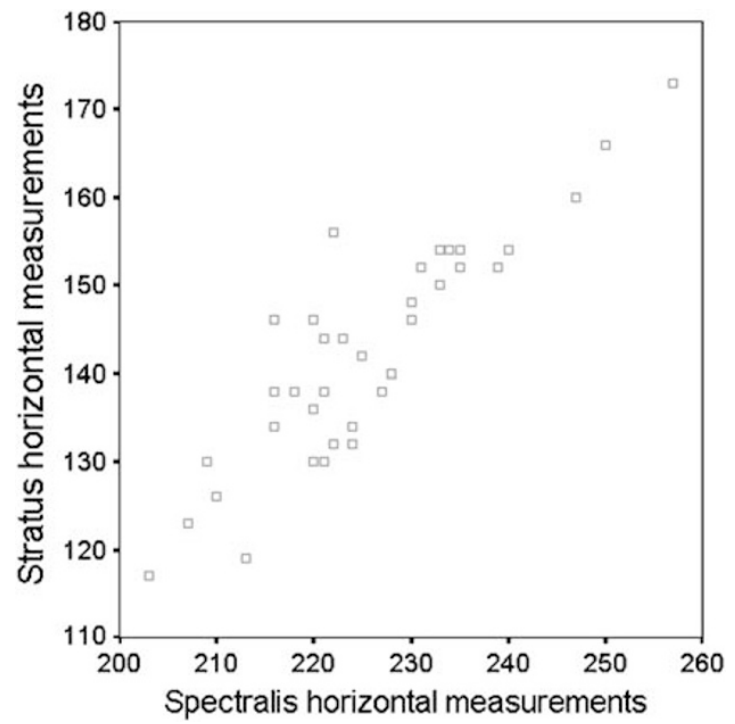

Figure 3 Linear relation between Spectralis and Stratus optical coherence tomography horizontal measurements. Pearson's correlation coefficient $(r)=0.899(P<0.001)$.

between two relatively high reflective structures represents the retinal thickness at that A-scan.

As a result, the OCT software locates the presumed inner retina boundary at the vitreo-retinal interface and the presumed outer retina boundary at the so-called 'RPEchoriocapillaris reflective complex' seen in first generations OCT. However, it has been recently shown that the above mentioned complex is composed of two well-defined highly reflective layers (HRL) at the level of the outer retina in the macular region of healthy participants: the inner HRL corresponding to the junction of the inner and outer segments of the photoreceptors,

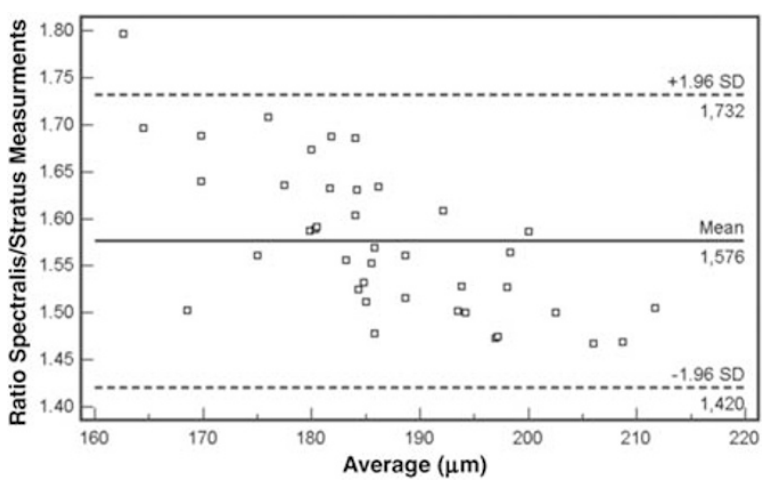

Figure 4 The Bland and Altman plot of the agreement in vertical foveal thickness between Spectralis and Stratus optical coherence tomography assessment. The ratio between Spectralis vertical foveal thickness and Stratus vertical foveal thickness is plotted against the average of the two measurements. Horizontal lines are drawn at the mean ratio, and at the mean ratio plus and minus 1.96 times the standard deviation (SD) of the ratios.

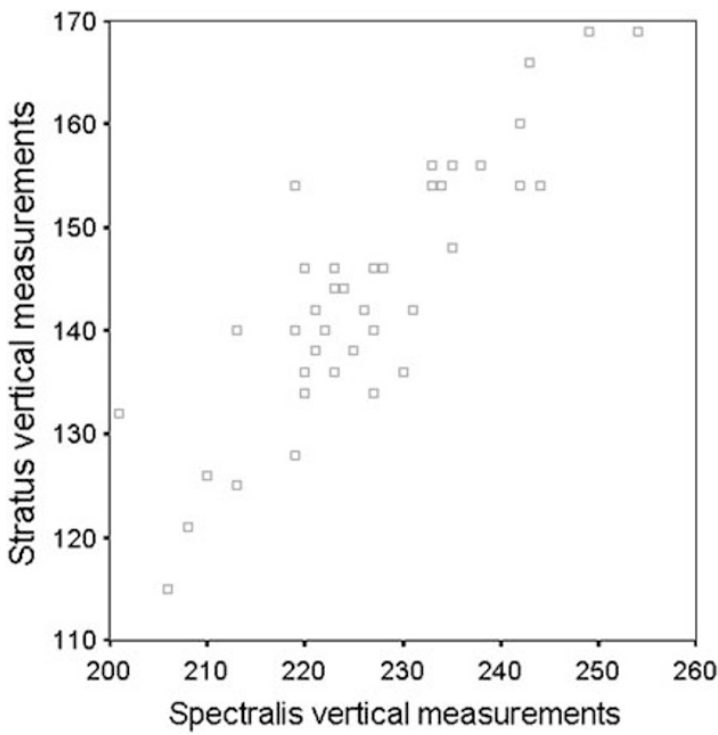

Figure 5 Linear relation between Spectralis and Stratus optical coherence tomography vertical measurements. Pearson's correlation coefficient $(r)=0.869(P<0.001)$.

and the outer one most likely corresponding to the retinal pigment epithelium or a reflective complex formed by RPE and choriocapillaris.

The use of the Stratus OCT automated retinal thickness measurement tool has been thought to generate erroneous values because of the incorrect interpretation of the inner HRL as the outer neural retina boundary. ${ }^{20,21}$ Manual caliper-assisted retinal thickness measurements, in which the outer HRL was interpreted as the outer boundary, at specific macular regions differed from those automatically generated by $9.9 \%$ from up to $38 \% .^{20}$ 
The Spectralis OCT thickness algorithm calculates retinal thickness as the distance between the vitreoretinal interface and the Bruch's membrane. Obviously, this generate different values from Stratus OCT being the measure of retinal thickness referring to two different anatomical section of the retina, also independently from the technical differences related to the detection systems of the two instruments.

Another topic arising from the study concerns the different variability of the results obtained with TD- and SD-OCT, using CoV to evaluate the degree of variation from one data series to another. Bauman et $a l^{22}$ tested the reproducibility of retinal thickness measurements of a prototype instrument of the first generation OCT, showing that mean CoVs ranged from 3.6 to $29.5 \%$ in the automated method and from 3.2 to $7.2 \%$ in the manually adjusted retinal thickness algorithm. Massin et $a l^{23}$ found mean CoVs ranging from 0.6 to $3.3 \%$ using the first commercially available OCT to test reproducibility of retinal thickness measurements in nine ETDRS areas of 10 healthy eyes.

Using Stratus OCT, Paunescu et al ${ }^{15}$ found mean central foveal thickness values ranging from 158.0 to $177.7 \mu \mathrm{m}$ with SDs 20.8 and $28.7 \mu \mathrm{m}$ for standard and high-density scanning, respectively, before and after dilation, in 10 normal participants. In this study $\mathrm{CoV}$ values can be estimated from 12.71 to $16.15 \%$. Later, Chan et $a l^{24}$ reported mean central foveal thickness values of $182 \pm 23$ and $170 \pm 18 \mu \mathrm{m}$ (automatically and manually determined, respectively) with $\mathrm{CoV}$ values of 12.64 and $10.59 \%$, respectively.

Similarly to our study, Leung et al $^{18}$ found lower CoV values when using SD-OCT (1.6-3.2\% for Stratus OCT; $0.6-2.4 \%$ for SD-OCT).

The lower degree of variation of results that we found when using Spectralis OCT may be related both to the high definition linked to the highest data acquisition rate and to a shorter examination time. As stated above, the motion artifacts in the Stratus OCT images require numerical correction, whereas the Spectralis OCT crosssectional image, measured in only $19 \mathrm{~ms}$, does not require any motion correction. In addition, higher scan resolution of SD-OCT allows a more detailed differentiation of retinal layers and more precise retinal border detection.

The Bland and Altman plot of the agreement in foveal thickness between Spectralis and Stratus OCT assessment showed the existence of a proportional bias for both horizontal and vertical scans.

Mean Spectralis/Stratus ratio for both horizontal and vertical measurements was 1.58 .

Correlation between the two different OCT techniques of foveal thickness measurement was not influenced by the amount of foveal thickness; in fact, Pearson's correlation indicated the existence of a linear relation between TD- and SD-OCT measurements.

Since the introduction of the OCT in the last decade, the ophthalmologists have gained new pathophysiological insights in a variety of ocular conditions. The OCT has become a fundamental tool for the diagnosis, treatment and follow-up of several macular and retinal diseases.

With the introduction of SD technology, a new generation of OCT devices has become available in the market. It is important to define the differences between the information disclosed by new devices as compared with the earlier known Stratus OCT, in order to properly introduce those new instruments into clinical practice, and to allow reliable comparison of data in patients examined with the two different methods.

This study analysed for the first time the differences between the foveal thickness measured by two different OCT models in healthy patients, comparing a new SD device with an earlier known TD-OCT. As expected, significant differences between the absolute values of foveal thickness assessed by the two OCT devices were detected. Nevertheless, a good correlation between Stratus and Spectralis OCT foveal measurements was found, independently of retinal thickness. This study suggests the existence of a conversion factor between Stratus and Spectralis OCT foveal thickness measurements and encourages the development of further investigation on larger samples, also including patients affected by macular pathologies.

\section{References}

1 Huang D, Swanson EA, Lin CP, Schuman JS, Stinson WG, Chang W et al. Optical coherence tomography. Science 1991; 254: 1178-1181.

2 Puliafito CA, Hee MR, Lin CP, Reichel E, Schuman JS, Duker JS et al. Imaging of macular diseases with optical coherence tomography. Ophthalmology 1995; 102: 217-229.

3 Wojtkowski M, Leitgeb R, Kowalczyk A, Bajraszewski T, Fercher AF. In vivo human retinal imaging by Fourier domain optical coherence tomography. J Biomed Opt 2002; 7: 457-463.

4 Nassif N, Cense B, Park BH, Yun SH, Chen TC, Bouma BE et al. In vivo human retinal imaging by ultrahigh-speed spectral domain optical coherence tomography. Opt Lett 2004; 29: 480-482.

5 Wojtkowski M, Bajraszewski T, Targowski P, Kowalczyk A. Real-time in vivo imaging by high-speed spectral optical coherence tomography. Opt Lett 2003; 28: 1745-1747.

6 Wojtkowski M, Bajraszewski T, czyñska I, Targowski P, Kowalczyk A, Wasilewski W et al. Ophthalmic imaging by spectral optical coherence tomography. Am J Ophthalmol 2004; 138(3): 412-419.

7 Chen TC, Cense B, Pierce MC, Nassif N, Park BH, Yun SH et al. Spectral domain optical coherence tomography: 
ultra-high speed, ultra-high resolution ophthalmic imaging. Arch Ophthalmol 2005; 123: 1715-1720.

8 de Boer JF, Cense B, Park BH, Pierce MC, Tearney GJ, Bouma BE. Improved signal-to-noise ratio in spectraldomain compared with time-domain optical coherence tomography. Opt Lett 2003; 28: 2067-2069.

9 Lumbroso B, Rosen R, Rispoli M. Principi e tecniche spectral OCT. In: Lumbroso B, Rosen R, Rispoli M (eds). Capire l'OCT spectral, Chapter 1 I.N.C: Rome, 2008; 9-10.

10 Frank H, Althoen SC. The coefficient of variation. In: Frank $\mathrm{H}$, Althoen SC (eds). Statistics: Concepts and Applications, Chapter 4.b Cambridge University Press: Cambridge, UK, 1995; 58-59.

11 Bland JM, Altman DG. Statistical methods for assessing agreement between two methods of clinical measurement. Lancet 1986; 1: 307-310.

12 Bland JM, Altman DG. Measuring agreement in method comparison studies. Stat Methods Med Res 1999; 8(2): 135-160.

13 Baumann M, Gentile RC, Liebmann JM. Reproducibility of retinal thickness measurements in normal eyes using optical coherence tomography. Ophthalmic Surg Lasers 1998; 29: 280-285.

14 Koozekanani D, Roberts C, Katz SE, Herderick EE. Inter-session repeatability of macular thickness measurements with Humphrey 2000 OCT. Invest Ophthalmol Vis Sci 2000; 41: 1486-1491.

15 Paunescu LA, Schuman JS, Price LL, Stark PC, Beaton S, Ishikawa $\mathrm{H}$ et al. Reproducibility of nerve fiber thickness, macular thickness, and optic nerve head measurements using stratus OCT. Invest Ophthalmol Vis Sci 2004; 45(6): 1716-1724.

16 Costa RA, Skaf M, Melo Jr LA, Calucci D, Cardillo JA, Castro JC et al. Retinal assessment using optical coherence tomography. Prog Retin Eye Res 2006; 25: 325-353.
17 Legarreta JE, Gregori G, Punjabi OS, Knighton RW, Lalwani GA, Puliafito CA. Macular thickness measurements in normal eyes using spectral domain optical coherence tomography. Ophthalmic Surg Lasers Imaging 2008; 39(Suppl 4): S43-S49.

18 Leung CK, Cheung CY, Weinreb RN, Lee G, Lin DS, Pang $\mathrm{CP}$ et al. Comparison of macular thickness measurements between time domain and spectral domain optical coherence tomography. Invest Ophthalmol Vis Sci 2008; 49: 4893-4897.

19 Forte R, Cennamo GL, Finelli ML, Crecchio GD. Comparison of time domain Stratus OCT and spectral domain SLO/OCT for assessment of macular thickness and volume. Eye 2008. [E-pub ahead of print 12 December 2008].

20 Costa RA, Calucci D, Skaf M, Cardillo JA, Castro JC, Melo Jr LA et al. Optical coherence tomography 3: automatic delineation of the outer neural retinal boundary and its influence on retinal thickness measurements. Invest Ophthalmol Vis Sci 2004; 45: 2399-2406.

21 Pons ME, Garcia-Valenzuela E. Redefining the limit of the outer retina in optical coherence tomography scans. Ophthalmology 2005; 112: 1079-1085.

22 Baumann M, Gentile RC, Liebmann JM, Ritch R. Reproducibility of retinal thickness measurements in normal eyes using optical coherence tomography. Ophthalmic Surg Lasers 1998; 29: 280-285.

23 Massin P, Vicaut E, Haouchine B, Erginay A, Paques M, Gaudric A. Reproducibility of retinal mapping using optical coherence tomography. Arch Ophthalmol 2001; 119: 1135-1142.

24 Chan A, Duker JS, Ko TH, Fujimoto JG, Schuman JS. Normal macular thickness measurements in healthy eyes using stratus optical coherence tomography. Arch Ophthalmol 2006; 124(2): 193-198. 